\title{
Comparative Study of Segmentation Techniques for Detection of Tumors Based on MRI Brain Images
}

\author{
Ashraf M. Said, Fatma S. Ibrahim* \\ Minia University /Biomedical Engineering, Minia, Egypt. \\ * Corresponding author. Tel.: 00201121627111; email: fatmasayed93@gmail.com \\ Manuscript submitted June 23, 2017; accepted September 4, 2017. \\ doi: 10.17706/ijbbb.2018.8.1.1-10
}

\begin{abstract}
The aim of this paper is to propose a matlab toolbox of a comparative study of four brain tumor segmentation methods with specific sequences. Four methods are K-means clustering, fuzzy C-means clustering, Region growing, and Otsu segmentation. Skull stripping algorithm has done before segmentation. Different preprocessing algorithms are applied before segmentation to enhance images and give better segmentation. Median filter, Gaussian filter, and contrast enhancement are applied according to segmentation method. The database used is based on DICOM images. GLCM used for feature extraction. Classification and accuracy measurement are done by ANN.
\end{abstract}

Key words: ANN, fuzzy C-means, GLCM, K-means clustering, otsu segmentation, region growing.

\section{Introduction}

The brain tumor is an abnormal mass of tissue in which cells multiply uncontrollably. Brain tumors can be primary or metastatic, and it further classified to be either malignant or benign. A metastatic brain tumor is kind of cancer which spread from another place in the body to the brain [1]. Imaging plays a vital role in the diagnosis of the brain tumors in earlier stages. Most common modalities that detect tumors are CT and MRI. MRI method is better than computer tomography due to its higher resolution, especially in brain imaging. Moreover, MRI imaging doesn't base on ionizing radiation so it's safer [2]. Image segmentation an important step in medical image analysis. Before segmentation varies techniques are done to enhance image quality and to prepare the image to be segmented. Preprocessing techniques depend on which segmentation method is performed. Varies methods have been proposed for the segmentation of brain tumor from MRI images. the paper successfully highlights the usefulness and limitations of threshold based, region based and pixel based automated segmentation techniques. Approaches are still not capable of being accepted among pathologist for everyday clinical tasks. Automatic diagnosis needs to be applied to all types of tumors as well as the necessity of standardized procedures [2]. In this paper, a comparison between K-means clustering, region growing, fuzzy c-mean and Otsu segmentation which based on the intensity threshold segmentation method are performed. Statistical data and features are extracted from an image using gray-level co-occurrence matrix (GLCM). In classification step, Tumors classified into benign and malignant using Artificial Neural Network (ANN). ANN used as accuracy measurement tool to figure out the best method. We found that K-mean gives higher accuracy and less elapsed time than the rest methods.

\section{Database}


Training dataset is collected from local radiology center with its reports that describe each image. Images used are in DICOM format and include normal brain images, grade one and grade two edemas. Brain tumor dataset are obtained from The Cancer Imaging Archive (TCIA) [3] which include astrocytoma and GBM brain tumors.

\section{Preprocessing}

A preprocessing stage considered to remove noise and enhance the quality of an image before segmentation. In this paper, varies preprocessing techniques are applied according to the corresponding segmentation method. Preprocessing techniques include skull stripping, median filter, Gaussian filtering and contrast enhancement.

\subsection{Skull Stripping}

Skull stripping is an important process in brain image analysis. It is a process of eliminating all non-brain tissues from brain image. skull stripping includes removing of extra-cerebral tissues such as skull's bone, fat, skin, etc. The proposal method is done by the

following sequence. First, Getting the dimensions of the image. Then checking color bands. If color bands are more than one, the image will be converted to grayscale. Second, Thresholding the image to create a binary image. Third, Removing of small specks of noise. Finally, seal off the bottom of the head and fill the image. "Fig. 1" illustrate the image before and after skull stripping.
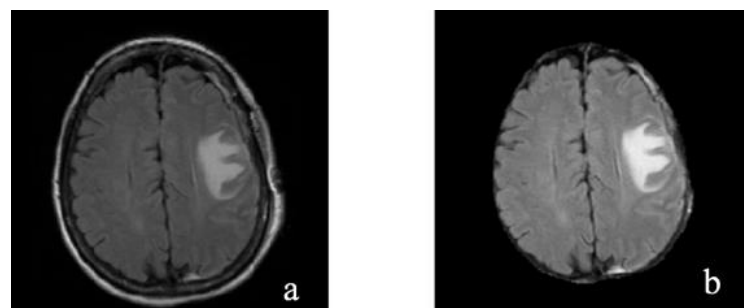

Fig. 1. (a) Original image, (b) Image after skull stripping.

\subsection{Median Filter}

Median Filter is used for noise reduction by removing salt and pepper noise. The main idea of the median filter is replacing each pixel with the average of neighboring pixels [4].

\subsection{Gaussian Filter}

The Gaussian smoothing operator is a convolution operator that is used to blur an image. Its kernel represents the shape of a Gaussian bell-shaped [5]. Gaussian filter can be expressed as

$$
I(X)=\frac{1}{2 \pi \delta^{2}} \cdot e^{-\frac{x^{2}+y^{2}}{2 \delta^{2}}} \cdot I(Y)
$$

\subsection{Contrast Enhancement}

If improves the image quality by enhancing hidden information. It is done by transforming the values in an intensity image, such that the histogram of the output image approximately matches a specified histogram. The output signal is of same data type as the input signal [6].

\section{Segmentation}

\subsection{K-Means Clustering}


K-means clustering technique is a pixel-based method. It is suitable for biomedical image segmentation as the number of clusters is known for images [4]. The results of it shown in "Fig. 2". K-means steps include following. Giving the number of cluster value as k. Randomly choose k cluster center $(\mu)$. Calculating mean or center of the cluster ( $\mu$ (i)). Calculating the distance between each pixel to each cluster center. If the distance is near, the center $(\mu=$ old $\mu$ ) then moving to that cluster. Calculating the distance between each pixel and each cluster center. Otherwise, move to next cluster. Re-estimating center. Repeating the process until the center doesn't move. Mathematical representation to compute the cluster means $\mu$ :

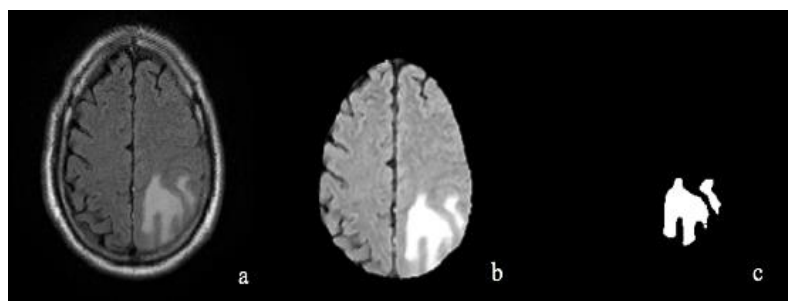

Fig. 2. (a) Original image, (b) Filtered image. (c) K-means segmentation.

$$
\mu=\frac{\sum_{i}^{n} X_{i}}{N_{k}}, k=1,2, \ldots \ldots . K
$$

where, $N k$ is number of clusters and $X i$ is data measured. Calculating the distance between the cluster centers to each pixel by following equation.

$$
D(i)=\min \left\|X_{i}-M_{k}\right\|^{2} \quad, i=1,2, \ldots N
$$

where, $X_{i}$ is the data measured, $M_{k}$ is the cluster centers [7].

\subsection{Region Growing Segmentation}

Segmentation implemented based on a set of seeds points, the grouping of pixels into regions based on seed points in which region grow by appending seeds to the neighboring pixels. The basic approach is to start with a set of "seed" points and from these grow regions by appending to each seed those neighboring pixels that have properties similar to the seed. Growing a region should stop when no more pixels satisfy the criteria for inclusion in that region [8]. "Fig. 3" illustrates the result of region growing segmentation. Proposed region growing segmentation is based on gray matter and white matter suppression. The basic formulations

$$
\begin{aligned}
& \cup_{i=1}^{n} R_{i}=R \text { where, } R_{i} \text { is a connected region } \mathrm{i}=1,2 \ldots \\
& \mathrm{Ri} \cap \mathrm{R} \mathrm{j}=\varnothing \text { for all } \mathrm{i}=1,2, \ldots, \mathrm{n} \\
& \mathrm{P}\left(R_{i}\right)=T R U E \text { for all } \mathrm{i}=1,2, \ldots ., \mathrm{n} \\
& \mathrm{P}(\mathrm{Ri} \cup \mathrm{Rj})=\text { FALSE for any adjacent region } \mathrm{R}_{\mathrm{I}} \text { and } \mathrm{R}_{\mathrm{j}}
\end{aligned}
$$

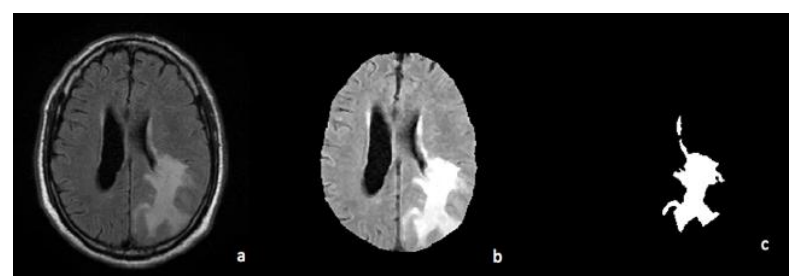

Fig. 3. (a) Original image, (b) Filtered image and (c) Region growing segmentation. 


\subsection{Otsu Segmentation}

Otsu's method, named after Nobuyuki Otsu is used to automatically perform clustering-based image thresholding. The part of the FLAIR MRI brain image containing the tumor normally has more intensity than the other portion. The algorithm assumes that the image contains two classes of pixels following bi-modal histogram (foreground pixels and background pixels), it then calculates the optimum threshold separating the two classes so that their combined spread (intra-class variance) is minimal, or equivalently [9]. We can define the within-class variance as the weighted sum of the variances of each cluster:

$$
\begin{gathered}
\sigma_{w}^{2}(I)=W_{f} \sigma_{f}^{2}(I)+W_{b} \sigma_{b}^{2}(I) \\
W=\frac{1}{n} \sum_{i}^{n-1} p(i)
\end{gathered}
$$

where, $\sigma_{w}^{2}(I)$ is within-class variance, $\sigma_{f}^{2}(I)$ the variance of the foreground $\sigma_{b}^{2}(I)$ is the variance of the background, $W_{f}$ the weight of the foreground $W_{b}$ the weight of the background, $P(i)$ is the intensity of the pixel and $(n)$ is a total number of pixels [9]. We found that Otsu segmentation is compatible with Gaussian filter preprocessing. "Fig. 4" shows the result of Otsu segmentation for four thresholds.

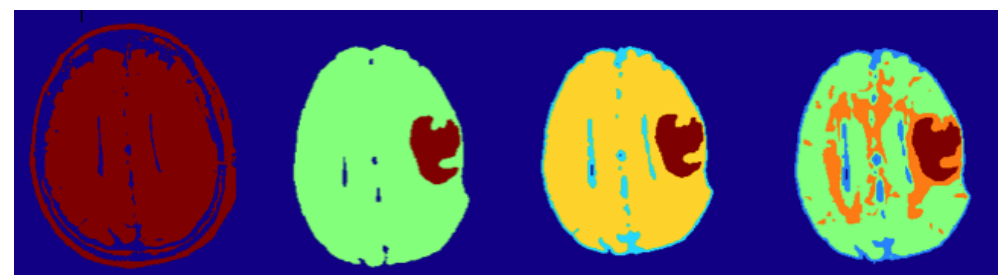

Fig. 4. Otsu segmentation for four thresholds.

\subsection{Fuzzy C-Means}

The fuzzy C-means algorithm is one of fuzzy clustering. FCM provides a high degree of membership to every pixel that is close to the threshold point [10]. The algorithm is powerful and more sophisticated than simple threshold algorithms. Hence, it carried out well in the segmentation of brain tumor images. It divides the brain texture into classes of tissue groups. The degree of relationship is assigned to pixels belonged in specific tissue class. the similarity between regions containing fuzzy membership functions in range 0 and 1 [10]. The partition should have two properties which are Homogeneity inside clusters and Heterogeneity between the clusters; the data, which belongs to different clusters. Fuzzy C-means steps:

Step 1: Choosing the number of clusters K.

Step 2: Set initial centers of clusters $\mathrm{c} 1, \mathrm{c} 2 \ldots \mathrm{ck}$.

Step 3: Classify each vector $\mathrm{x}[\mathrm{x} 1, \mathrm{x} 2, \ldots \mathrm{xn}]$ into the closest center ci by distance measure $\|\mathrm{xi}-\mathrm{ci}\|=$ $\min || x i-c i||$.

Step 4: Recomputed the estimates for the cluster centers ci Let $\operatorname{ci}=[\operatorname{ci} 1, \operatorname{ci} 2, \ldots . . \operatorname{cin}] \mathrm{T}$, cim be computed by:

$$
c_{i m}=\frac{\sum x l i \in \text { Cluster(Ixlim) }}{N_{i}}
$$

where $\mathrm{Ni}$ is the number of vectors in the ith cluster.

Step 5: If none of the cluster centers $(c i=1,2, \ldots, k)$ changes in step 4 stop; otherwise go to step 3 [11]. 


\section{Feature Extraction}

A statistical method of examining texture that considers the spatial relationship of pixels are the gray-level co-occurrence matrix (GLCM), also known as the gray-level spatial dependence matrix. The GLCM functions characterize the texture of an image by calculating how pairs of a pixel with specific values and in a specified spatial relationship occur in an image, creating a GLCM. This new matrix used to extract statistical measures [4]. "Fig. 5" shows the concept of GLCM and how the new matrix is generated. New matrix shows the distribution of the gray levels in the image. Twenty-two statistical features are derived from the new matrix. Statistical features include contrast, correlation, autocorrelation, energy, homogeneity, variance and etc.

\begin{tabular}{|c|c|c|c|c|c|c|c|}
\hline & & & GLCM & 1 & 2 & 3 & 4 \\
\hline 1 & $1)$ & 4 & 1 & $\rightarrow 1$ & 0 & 2 & 1 \\
\hline 2 & 1 & 3 & 2 & 1 & 0 & 0 & 0 \\
\hline 1 & 3 & 2 & 3 & 0 & 1 & 0 & 0 \\
\hline & nal me & & 4 & 0 & 0 & 0 & 0 \\
\hline
\end{tabular}

Fig. 5. The process of creation the GLCM.

\section{Classification Using ANN}

A neural network can be defined as a model of reasoning based on the human brain [12]. In this work, we use back propagation neural network (BPNN) with ten hidden layers. BPNN consists of components connected together which mimic the biological neurons. Each neuron in ANN has many inputs and one output given by applying activation function. "Fig. 6" shows simple neural network architecture.

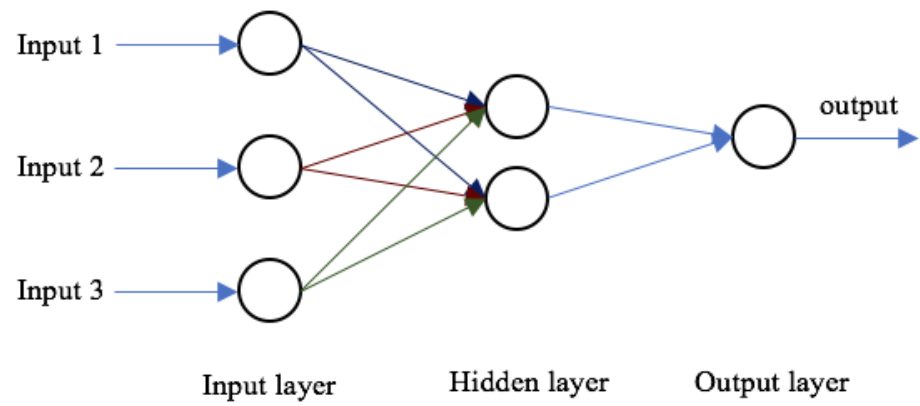

Fig. 6. Simple neural network architecture.

BPNN consist of four stages. First, Initialization of weights. Second, Feed forward. Third, Back propagation of error. Fourth, Updating of weights and biases. The input of NN in this case is a matrix of the twenty-two features for all training dataset and the output is a matrix that classify all images into their classes. ANN used as a tool for classification and accuracy measurement in our comparative study. The network was trained and its performance was evaluated. The comparative study is performed and the results of reached technique are calculated. "Table 1" shows the results of grade 1 tumors in the first row and grade 2 tumors in the second row. The performance of ANN classifier described by confusion matrix. ANN confusion matrix for best results with an accuracy of 96.7\% shown in "Fig. 7". 
Table 1. Comparison Result of Segmentation with Its Accuracy

\begin{tabular}{|l|l|l|l|l|l|}
\hline $\begin{array}{l}\text { Original MRI } \\
\text { image }\end{array}$ & Filtered image & K-means & Region growing & $\begin{array}{l}\text { Otsu } \\
\text { segmentation }\end{array}$ & FCM \\
\hline & & & & & \\
\hline & & & & & \\
\hline
\end{tabular}

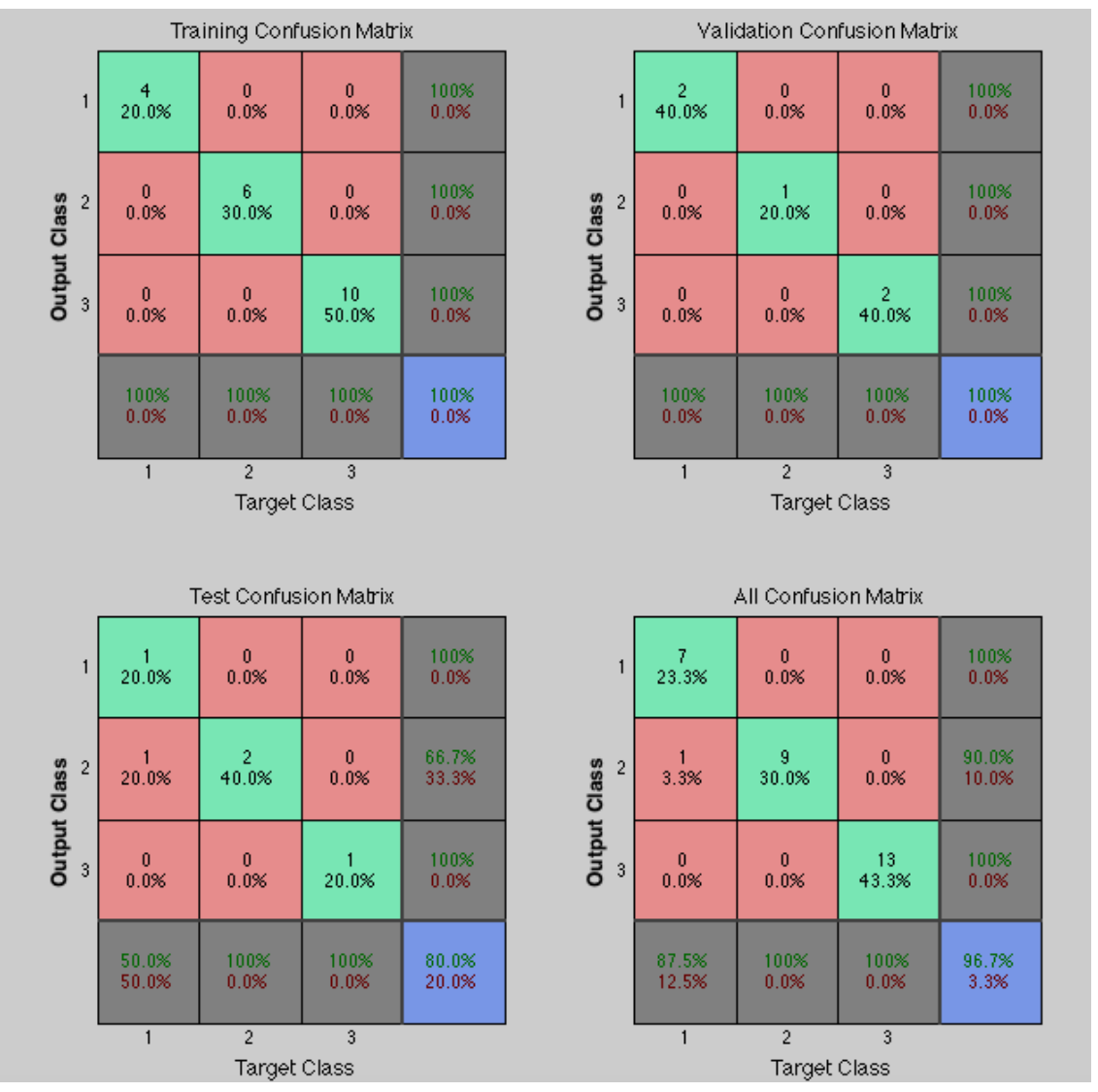

Fig. 7. ANN evaluation using confusion matrix.

\section{Sequences and Results}

As shown in the previous sections we propose a sequence for each segmentation. The result of 
segmentation is depending on what filters are applied. segmentation process segment brain tumor from an image. All sequences are organized in the same platform and introduced in the graphic user interface (GUI) is shown in "Fig. 8 (a)". The proposed algorithm, which based on K-means for segmentation, GLCM as a feature extraction and back propagation network classifier has been successfully tested and achieved the best results with accuracy $96.7 \%$. with 2.2 seconds elapsed time. The first sequence is illustrated in "Fig. 8 (b)" which gives the best result and highest accuracy.
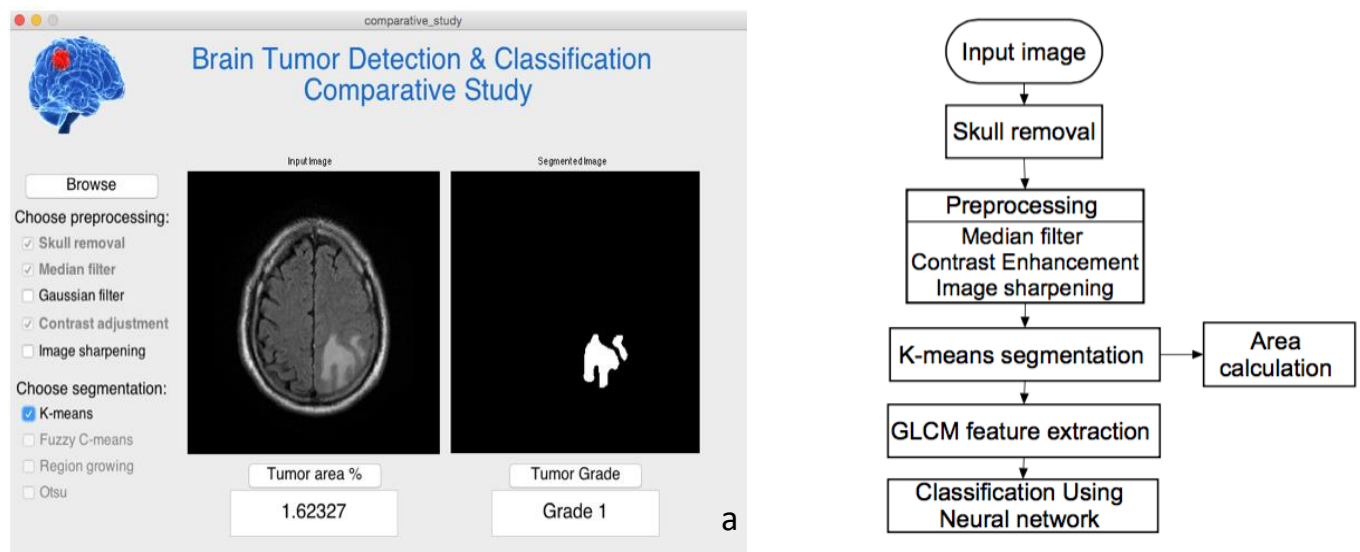

Fig. 8. (a) GUI for toolbox of all methods used in comparative study applying first sequence. (b) The first sequence for detection and classification of brain tumor.
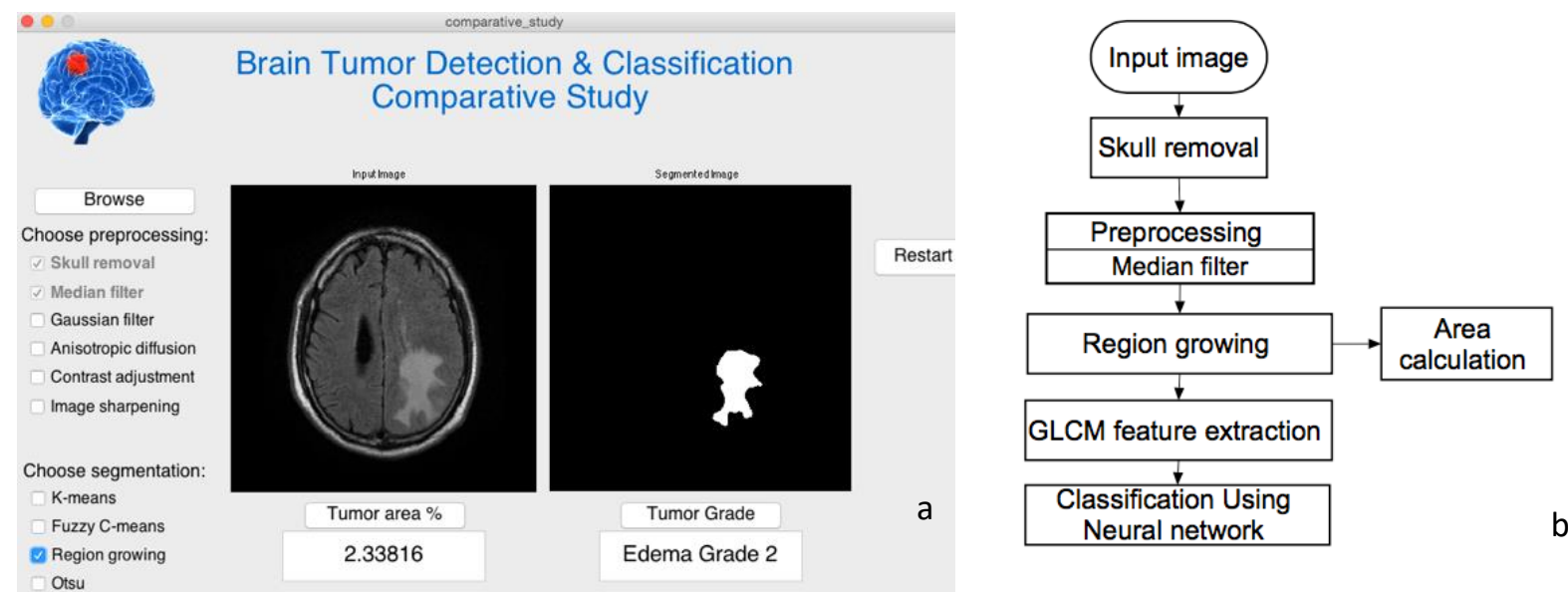

Fig. 9. (a) GUI for second sequence for detection and classification of brain tumor. (b) The second sequence for detection and classification of brain tumor.
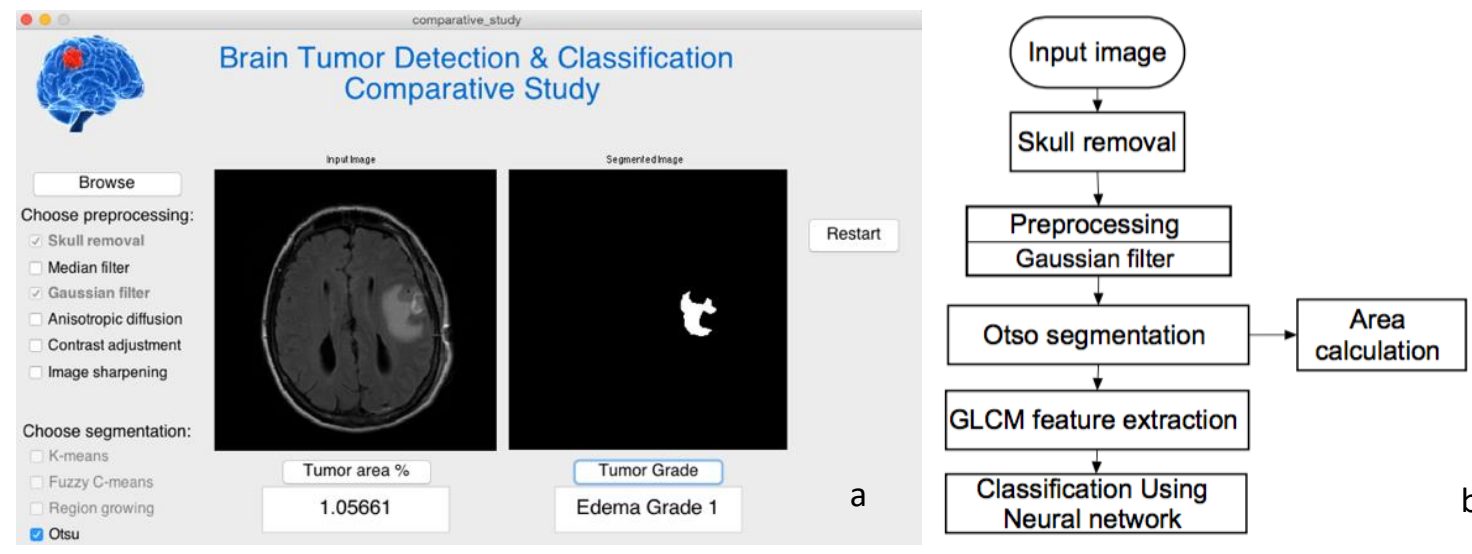

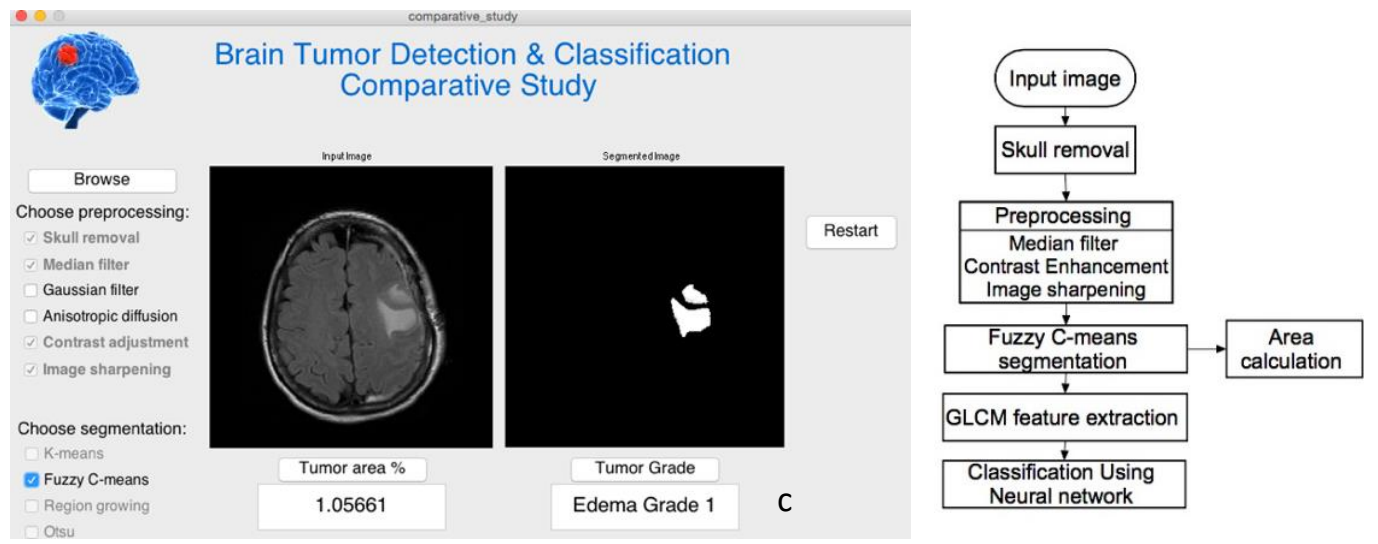

Fig. 10 (a) GUI for third sequence. (b) Flow chart of the third sequence. (c) GUI for fourth sequence. (d) Flow chart of the fourth sequence.
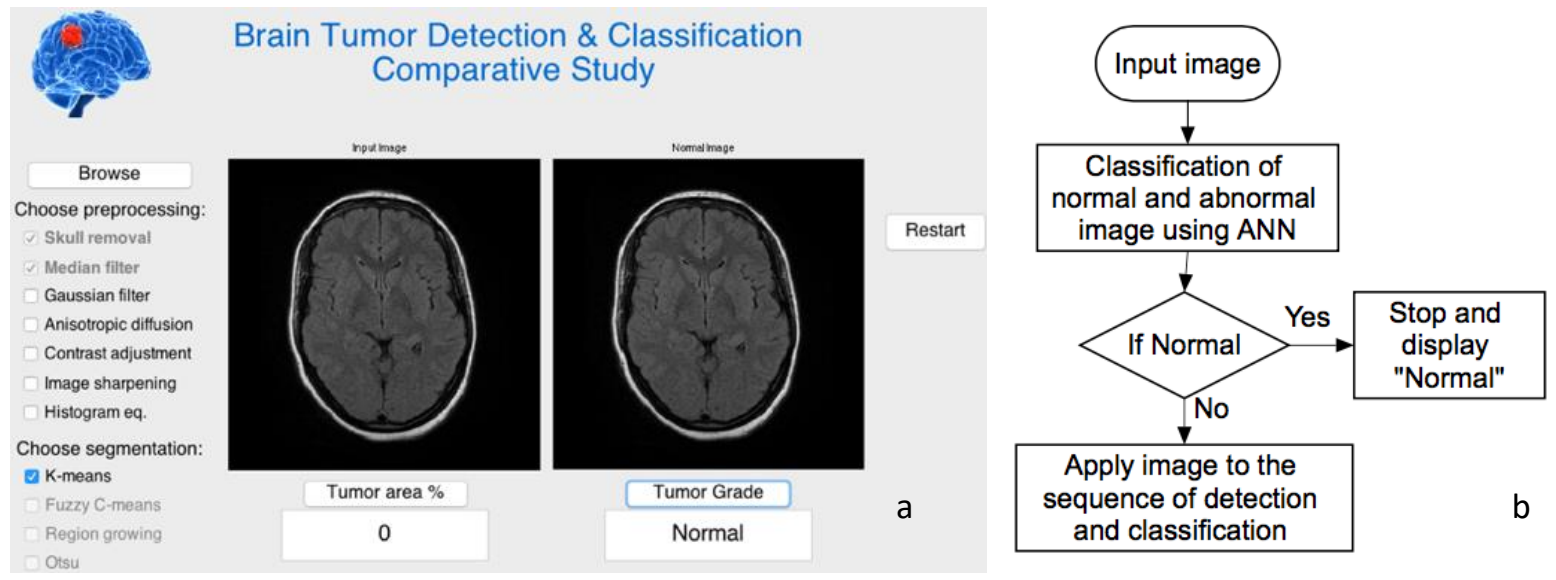

Fig. 10. (a) GUI detects normal brain image. (b) Flow chart for detection of normal images.

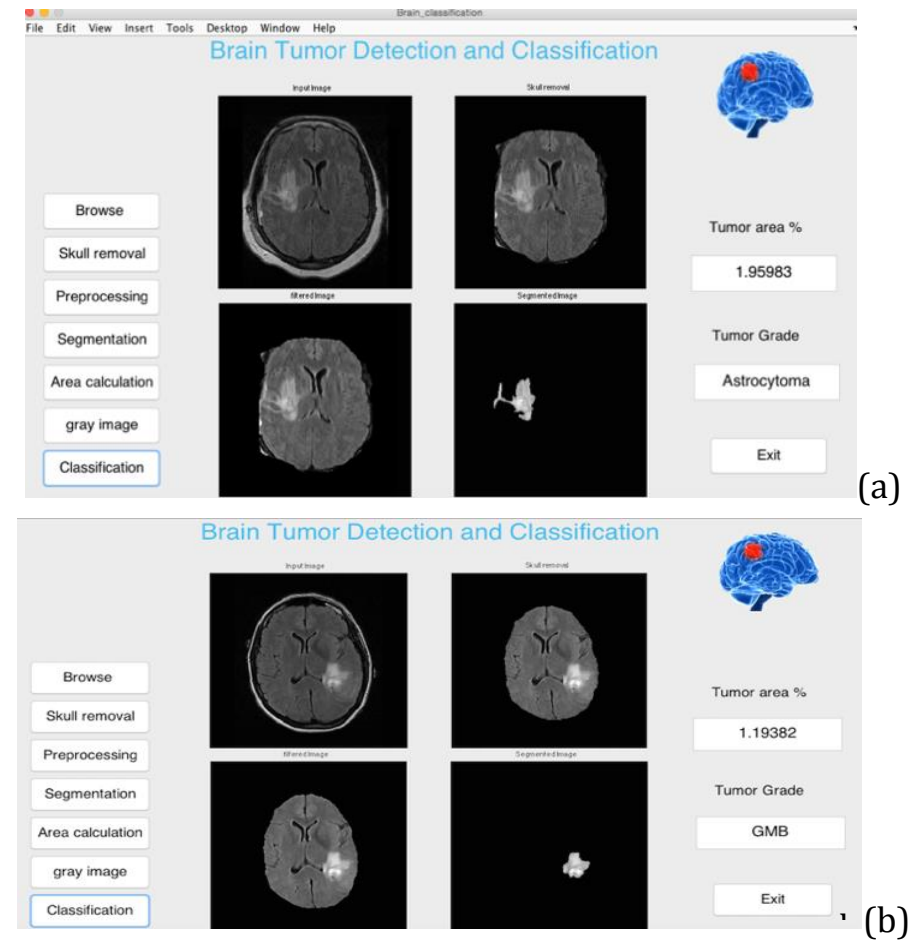

Fig. 11. (a) GUI detect astrocytoma tumor (b) GUI detects GMB tumor. 
The combination of median filter, contrast enhancement, and image sharpening makes the intensity of each part of brain good separated and then make the result of k-means segmentation more accurate. The second sequence which apply median filter and region growing segmentation has the lowest accuracy and longer elapsed time for execution. "Fig.9 (a)" shows the result of second sequence in GUI. "Fig. 9 (b)" shows the flowchart for second sequence.

In the third sequence, we apply a Gaussian filter which smooths the image and make it easy to apply Otsu segmentation. This sequence gives high accuracy reach 90\%. "Fig. 10 (a)" shows the result of the third sequence in GUI. "Fig. 10 (b)" shows the flowchart for the third sequence. The fourth and last successful sequence is based on the median filter, contrast enhancement and image sharpening as preprocessing and fuzzy C-mean as a segmentation. This sequence gives high accuracy and its results are similar to the first sequence. The back draw of this sequence is its prolonged elapsed time.

The normal images are detected before introducing image to the sequence of detection and classification of a tumor. "Fig. 11 (a)" shows the result of normal image. "Fig. 11 (b)" shows the flow chart of detection normal image.

The first sequence gives promised results in detection and classification of astrocytoma and glioblastoma tumors. "Fig. 12 (a)" shows astrocytoma detection and "Fig. 12 (b)" shows GMB detection.

\section{Conclusion}

Extraction of tumor region is an important step in diagnosis and treatment planning. Despite the computer aided techniques are sophisticated and require enormous effort to be implemented but not as manual methods which are time-consuming and can undergo to human errors. The core of the proposed implementation is to introduce four image segmentation methods that have been implemented based MR image dataset in DICOM format. GLCM features are taken from images after segmentation. ANN Classify tumors and used as a measurement tool to compare among segmentation methods. Different sequences of segmentation have been introduced in one toolbox. It is the first time to organize varies pre-processing and segmentation techniques in one graphical interface.

Astrocytoma and GBM have been discriminated and classified using different recognition sequences. these sequences inferred and recognized the types of tumors in different accuracies according to results of propose implementations. The results of ANN prove that K-means method is found to be the best segmentation method among the other four segmentation methods which implemented. K-means method found to be the best because of the nature of MRI FLAIR image and the brain tumor images which consists of four different classes; The tumor, white matter, gray matter and cerebrospinal fluid (CSF) which appear black in the image as the background. K-means show accurate results with very short elapsed time. In Future, we want to obtain the safety boundary of the tumor and plotting its margin for surgery planning. This will help doctors to make surgery based on automatic boundary calculation. Furthermore, protect the patient from further biopsy procedure. We are planning to propose a complete system with brain mapping of EEG to detect abnormalities with higher accuracy.

\section{Acknowledgment}

Appreciation and thanks to everyone supported this work for his assistance and guidance. Special thanks to the local radiology center for their cooperation that greatly improves our research.

\section{References}

[1] Bhattacharyya, D., \& Kim, T. (2011). Brain Tumor Detection Using MRI Image Analysis (pp. 307-314).

[1] Qadar, M. A., \& Zhaowen, Y. Brain Tumor Segmentation : A Comparative Analysis. 
[2] Cancerimagingarchive. Retrieved from the website: http://www.cancerimagingarchive.net/

[3] Student, U. G. (2014). Feature Extraction of Brain Tumor Using MRI, 3(3), 10281-10286.

[4] Gonzalez, R. E. W., \& Hall, P. Digital Image Processing.

[5] Khawaja, A. (2015). Contrast Enhancement Impact on Detection of Tumor, 27(3), 2161-2163.

[6] Patil, A. J., et al. (2005). Pachpande, Automatic Brain Tumor Detection Using K-Means, 13896-13903.

[7] Tanuja, M., \& Shewale, P. (2016). Detection of Brain Tumor Based On Segmentation Using Region Growing Method, 5(2), 173-176.

[8] Otsu, N. (1979). A threshold selection method from gray-level histograms. IEEE Transactions on Systems, Man, and Cybernetics, 9(1).

[9] Bhide, P. A. S. (2012). Brain Segmentation Using Fuzzy C means Clustering to Detect Tumour Region, 1(2), 85-90.

[10] Suganya, R., \& Shanthi, R. (2012). Fuzzy C- Means Algorithm - A Review, 2(11), 1-3.

[11] Kochar, P. (2014). A Survey on Brain Tumor Detection and Classification system based on Artificial Neural Network, 90(18), 16-21.

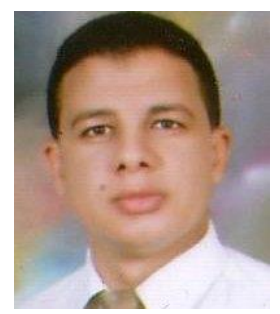

Ashraf M. Said graduated from biomedical engineering in Cairo University, his master degree, M.Sc. in optimum design of MRI gradient and RF coils in 2005 while Ph.D. is the statistical analysis of Brian connectivity using Bold images of FMRI on human vision system, the Ph.D. graduation in 2011, He is currently an Assistant Professor in Biomedical Engineering department in Faculty of engineering in Minia University. His scope of research interests in Bio signal processing, recognition in biometric, Image processing, biotechnology, and biomedical application of nanotechnology

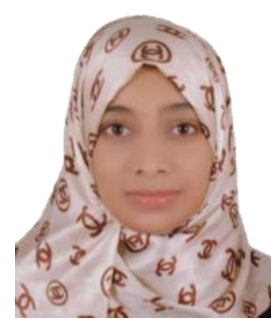

Fatma S. Ibrahim received the B.S. degrees in biomedical engineering from the Minia University, Egypt, in 2016. She is currently a teaching assistant in Minia University. Her research interests include structural and functional MR image analysis, embedded system, medical imaging and neural networks. 